Historic, Archive Document

Do not assume content reflects current scientific knowledge, policies, or practices. 



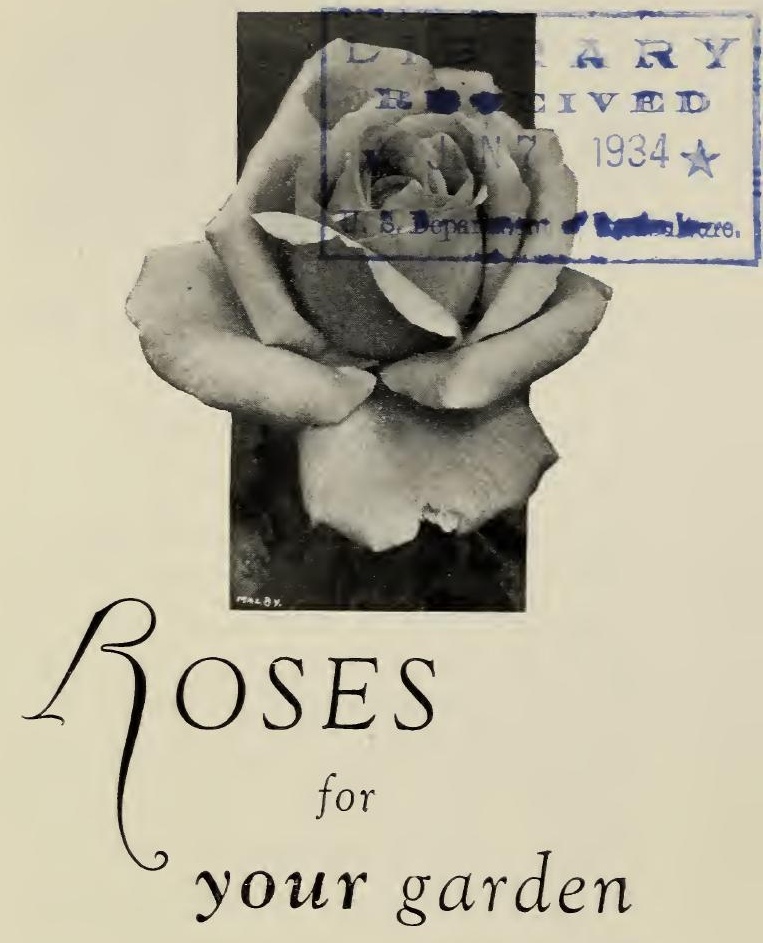

WAITE'S GARDENS INC.

EATONTOWN NEW JERSEY 
I PRESENTING this little rose folder, we have endeavored to congregate in concise form, a collection of varieties that will thrive under ordinary conditions, and with a little extra care and attention will give much pleasure and satisfaction to the planter, with their large blooms of most delightful colors, - an uninterrupted succes. sion of floral splendour from June until October.

We guarantee our plants to be true to name and healthy, all 2-year old, low-budded, fieldgrown plants, but we will not replace except where mislabeled.

As we have no control over subsequent treatment after leaving our grounds, the buyer must assume all risks thereafter, whether planted by us or not.

\section{HYBRID TEA}

\section{or Everblooming Roses}

Prices: 85c. each, except where priced otherwise.

Admiral Ward. Brilliant crimson-red; deliciously fragrant; prolific bloomer.

Apotheker Geo. Hoefer. Bright carmine: large full flowers.

Betty. Coppery rose, overspread with golden yellow.

Briarcliff. Long pointed buds, and full flowers of a light rose pink; each $\$ 1.00$.

Chateau de Clos Vougeot. Striking velvety-scarlet turning to deep crimson. Free bloomer; each $\$ 1.00$.

Columbia. Large full flowers, deep rose pink.

Duchess of Luxemburgh. Brilliant coppery red; splendid bloomer; each $\$ 1.00$.

Duchess of Wellington. Saffron-yellow, stained crimson; each $\$ 1.00$.

Edel. A rose of exhibition quality. White flowers that open ivory-white.

Elvira. A splendid flower of rich rose pink; each $\$ 1.00$.

Etoile de France. Soft velvety crimson shaded cerise in center.

Francis Scott Key. Blazing red, large, perfectly formed flowers.

General-Superior A. Janssen. Fragrant blooms of light crimson-carmine.

Gruss an Teplitz. Gives clear crimson-scarlet flowers. 


\section{HYBRID TEA ROSES}

\section{Continued}

Hadley. Large crimson-scarlet blooms.

Jonkheer J. L. Mock. Cherry red, silvery reverse; very large.

Kaiserin Augusta Victoria. Large, full creamy white blooms; each $\$ 1.00$.

Killarney Brilliant. A much improved "Killarney", with more color.

Killarney Queen. A distinct, clear pink.

Killarney, Double White. Similar to the famous "Killarney" in size; snow white.

Lady Alice Stanley. Deep coral-rose, pale flesh on inside.

Lady Ashtown. Pale carmine-pink shading to golden yellow.

Lady Hillingdon. Clear apricot-yellow flowers; each $\$ 1.00$.

Los Angeles. Flame-pink toned with coral and yellow at base; each $\$ 1.00$.

Louise Catherine Breslau. Coral red, shaded chrome-yellow; each $\$ 1.00$.

Mme. Butterfly. Brilliant pink suffused apricot.

Mme. Caroline Testout. Broad petals of satiny rose.

Mme. Edouard Herriot. Coral red buds, developing into flowers of the same hue.

Mme. Jules Bouche. White flowers, shaded prim. rose.

Mme. Jules Grolez. Bright china-rose blooms of good size.

Mme. Leon Pain. Salmon, shaded to orangesalmon.

Mme. Aaron Ward. Deep Indian Yellow, varied and shaded orange.

Mrs. Chas. Bell. Beautiful blossoms of delicate shell-pink; each $\$ 1.00$.

Mrs. Franklin Dennison. Porcelain white, slightly shaded primrose yellow.

Mrs. Mackellar. Delicate light yellow with creamy buds.

Ophelia. Light salmon-flesh color, shading to yellow.

Radiance. Salmon pink suffused coppery red Very prolific.

Red Radiance. Similar in growth and habit to preceding, only deep red. 


\section{HYBRID TEA ROSES}

Continued

Rev. F. Page-Roberts. Beautiful Marechal Niel yellow, veined with buff; each $\$ 1.00$.

Rose Marie. Clear, bright rose-pink, with lighter edge.

Souv. de Claudius Pernet. A most vigorous grower; clear rich yellow; each $\$ 1.00$.

Souv. de Georges Pernet. Rich red shaded co. chineal-carmine; each $\$ 1.00$.

Souv. de President Carnot. Rosy flesh, shaded white at edges.

Wm. R. Smith. Soft silvery white shaded pink.

Willowmere. Coral red buds, later rich shrimppink; each $\$ 1.00$.

\section{CLIMBING ROSES}

The most ornamental of all Climbing Vines for Porch, Ttellis or Arbor. Prices: each 85c.; except where specially priced.

Alberic Barbier. Buff yellow and creamy white.

American Pillar. Light, bright crimson with white eye.

Aviateur Bleriot. E::quisite saffron-yellow, shaded apricot.

Climbing American Beauty. Rich carmine color.

Dorothy Perkins. Soft blush pink, free bloomer.

Dr. Van Fleet. Deep, clear pink, becoming lighter.

Electra. Double yellow and creamy flowers.

Emily Gray. Best yellow climber. Foliage glossy green; each $\$ 1.00$.

Heart of Gold. Crimson with central zone of golden yellow stamens.

Mary Wallace. Very large, clear rose-pink; each $\$ 1.00$.

Paul's Scarlet Climber. Clear, vivid, scarlet flowers; never turns pale.

Silver Moon. Faint yellow buds, opening to pure white.

Star of Persia. Bright yellow; a wonderful pillar rose.

Tausendschon. Buds first open cherry-pink changing to lighter shade.

White Dorothy Perkins. Just like "Dorothy Perkins" only white. 